\title{
Inflammatory Bowel Disease Management in a Romanian Tertiary Gastroenterology Center: Challenges of the COVID-19 Pandemic
}

\author{
Roxana Vadan ${ }^{1}$, Razvan Iacob ${ }^{1,2}$, Roxana Costache ${ }^{1}$, Tudor Stroie ${ }^{1}$, Adrian Ionut Saizu ${ }^{1}$, Speranta Iacob $^{1,2}$, Liana \\ Gheorghe $^{1,2}$, Mircea Diculescu ${ }^{1,2}$, Cristian Gheorghe ${ }^{1,2}$
}

1) Digestive Diseases and Liver Transplantation Center, Fundeni Clinical Institute, Bucharest;

2) Carol Davila University of Medicine and Pharmacy, Bucharest, Romania

\author{
Address for correspondence: \\ Razvan Iacob MD, $\mathrm{PhD}$ \\ Digestive Diseases and Liver \\ Transplantation Center \\ Fundeni Clinical Institute \\ 258 Fundeni Str., Sector 2 \\ 022328, Bucharest, Romania \\ raziacob@gmail.com
}

\begin{abstract}
Background \& Aims: Inflammatory bowel diseases (IBD) patients management has been challenging during the ongoing coronavirus disease 2019 (COVID-19) pandemic, due to lockdowns, limitation of access to medical facilities and new recommendations regarding patient management. The implications of the COVID-19 pandemic on IBD patients' management were assessed in our Tertiary Gastroenterology Center in Bucharest, Romania.

Methods: Medical records of IBD patients admitted between 15th of March and 15th of August 2020 were retrospectively reviewed and compared to a control cohort of consecutive IBD patients admitted to our unit during the corresponding period of 2019.

Results: There was a highly significant shift towards one-day hospitalization during the referral period in 2020 for IBD cases ( $91 \%$ in 2020 vs $82.2 \%$ in $2019, \mathrm{p}=0.0001$ ). There was no statistically significant difference between the distribution of patient's gender, IBD phenotype or newly diagnosed IBD cases. A significantly lower proportion of admitted patients received 5 -aminosalicylic acid ( $29 \%$ vs $41.2 \%$, p=0.0001), whereas a substantially higher number of patients were prescribed biological therapy in 2020 in comparison to the corresponding 2019-time frame (79.5\% vs 57.9\%, p<0.0001). The distribution of the biological agent used was significantly different in 2019 in comparison to the 2020 period mainly due to the increase in vedolizumab prescription in 2020 ( $\mathrm{p}<0.0001)$. During the study period in 2020, seven IBD patients $(1.7 \%)$ were diagnosed with severe acute respiratory syndrome coronavirus 2 (SARS-Cov2) infection, all of them with mild symptoms without impact on the IBD course.

Conclusions: The COVID-19 pandemic led to reorganizing medical care, limiting the hospital admissions in favor of severe IBD cases, favoring telemedicine for mild disease and optimization of treatment for moderate to severe IBD with an increased use of biologicals aimed to maximize the risk/benefit ratio. Incidence of SARS-Cov2 infection during the first wave of COVID-19 infection in our study group was $1.7 \%$ and did not adversely impact the IBD disease course.
\end{abstract}

Key words: inflammatory bowel diseases - biological therapy - COVID-19 pandemic - Romania.

Abbreviations: ACE2: angiotensin converting enzyme 2; AZA: azathioprine; CD: Crohn's disease; COVID-19: coronavirus disease 2019; CS: corticosteroids; IBD: inflammatory bowel diseases; PCR: polymerase chain reaction; RGD: arginine-glycine-aspartate; SARS-Cov2: severe acute respiratory syndrome coronavirus 2; UC: ulcerative colitis; 5-ASA: 5-aminosalicylic acid.

\section{INTRODUCTION}

The coronavirus pandemic, responsible to date for over 62 million infected people all over the world and over 1.4 million coronavirus related deaths is posing great challenges to medical systems [1]. Since the first patient with coronavirus disease 2019 (COVID-19) in
Romania confirmed on February 26, 2020 in the southern part of the country, the disease has spread widely, with over 465.000 cases and over 11,000 fatalities reported by the end of November 2020. Subsequent to the WHO Declaration of the coronavirus pandemic in March 2020, the medical care has been reorganized in Romania, to cope with the increasing number of COVID-19 cases. There have been designated COVID-19 medical care units and COVID-19 support hospitals that were exclusively dedicated to the medical assistance of COVID-19 patients, with or without comorbidities. This reorganization has supported the effort to keep the main hospital workforce 
and the severe acute respiratory syndrome coronavirus 2 (SARS-Cov2)-negative patients free of COVID-19 and thus, to allow regular medical activity to continue. Our unit, a Tertiary Gastroenterology Referral Center and Inflammatory Bowel Diseases (IBD) Center of Excellence is involved in the medical care of SARS-Cov2 negative patients. Changes in our medical activity were implemented, to comply with the Romanian Health Ministry recommendation, supported by the Romanian Society for Gastroenterology and Hepatology and Romanian Society for Digestive Endoscopy to suspend all elective endoscopic or surgical procedures and also nonurgent admissions during the 2 months lock-down period, from March until May 2020, with the aim to mitigate the risk of spreading COVID-19, until adequate hospital reorganization was in place $[2,3]$. We had to quickly adapt and find ways to optimize patient care in this new medical setting, to find a balance between social distancing and the need to provide the required medical care. As an IBD Referral Unit mandatory admissions for IBD flares, time sensitive appointments for biological therapy and addressed patients' and caregivers' concerns regarding risk of exposure during travel to hospital or during hospitalization and of potential risks associated with IBD treatment on the severity of SARS-Cov2 infection have been permitted. Our activities were in line the preoccupations of the worldwide of worldwide gastroenterological community involved in the management of IBD cases, trying to prevent, promptly diagnose and manage of COVID-19 in IBD patient, leading to a favorable course of the disease [4-6].

In the present study we have evaluated the impact of the COVID-19 pandemic on IBD patients management in our tertiary gastroenterology unit. We have also reported the incidence and outcome of IBD patients from our unit diagnosed with SARS-Cov2 infection during the study period.

\section{METHODS}

Hospital computerized registries were reviewed comparing a 5-month period (15th of March - 15th of August) in 2020 to the corresponding time frame in 2019. In 2020 this referral period included the two months general lockdown period (16th of March to 16th of May) that comprised significant modifications in patient referral and management. The main aspects concerning patients management in 2020, especially during the general lockdown consisted of the implementation of online consultations and hospital admission only with prior appointment after the online consultation with the attending physician, or for emergencies, as well as other significant epidemiological measures. A hospital check-point pre-hospitalization in all patients referred to our Unit (temperature check, presence of COVID-19 symptoms, infected person contact tracing, travel history) was implemented. General hygiene measures were reinforced: reorganizing the ward setting to accommodate an in-patient triage area for COVID-19, reorganizing the dedicated area for biological treatment administration, re-enforcing rigorous preventive measures for medical staff and patients - adequate personal protective equipment wearing for medical staff, hand hygiene, patients mask wearing. Strict spacing requirement for hospitalized patients mandating physical distancing were implemented and SARS-Cov2 polymerase chain reaction (PCR) testing was performed for all admitted cases and prior to all endoscopic examinations. Overall, telemedicine and online consultations were used for individual case management, with a shift towards primary care management whenever possible. Routine endoscopies were postponed, and only mandatory and emergent procedures were conducted.

All hospital charts of IBD patients admitted during the two referral periods were reviewed and significant epidemiological and clinical data was registered in a dedicated excel worksheet, with defined variables. Statistical analysis was conducted using NCSS Statistical Software package v9.0.7. Categorical data is depicted in the tables as the number of cases in the respective categories and percentage of cases and was compared using the chi-square test or Fisher Exact test. Continuous data was compared between the groups using the Student's T test and the Mann Whitney U test, when appropriate, with a two-tailed $p$ value $<0.05$ considered for statistical significance.

Descriptive statistics were provided for patients with IBD tested positive for SARS-COV2 infection in our unit during the study period, by the means of a PCR test from a nasopharyngeal swab. All COVID-19 positive patients were contacted by phone to obtain the data regarding the severity and outcome of viral infection (discharge letters from COVID-19 hospitals, documenting disease symptoms and treatments).

\section{RESULTS}

There was a highly significant shift towards one-day hospitalization during the referral period in 2020 for IBD cases: 1,059 admissions (9\% ward hospitalizations, $91 \%$ one-day hospitalizations) compared to 1,327 cases in the corresponding period of 2019 (17.8\% ward hospitalizations, $82.2 \%$ one day hospitalizations $)(\mathrm{p}<0.0001)$. Overall, 410 individual IBD cases were managed in the hospital IBD unit setting in 2020 compared to 532 cases in 2019.

Patients characteristics are depicted in Table I.

There was no statistically significant difference between the distribution of patient's gender, IBD phenotype or newly diagnosed IBD cases between the two periods. However, the

Table I. Comparison of IBD patient's characteristics during the two study periods (2019 vs 2020)

\begin{tabular}{lccc}
\hline & 2019 & 2020 & $\mathrm{p}$ \\
\hline IBD admissions, N & 1327 & 1059 & $\mathrm{NA}$ \\
$\quad$ All IBD admissions & 532 & 410 & $\mathrm{NA}$ \\
IBD individual cases & & & \\
Gender, N (\%) & $278(52.2)$ & $218(53.2)$ & 0.83 \\
$\quad$ Male & $254(47.8)$ & $192(46.8)$ & \\
$\quad$ Female & $43.7 \pm 14.7$ & $41 \pm 14.2$ & 0.006 \\
Age, years \pm SD & & & \\
IBD phenotype, N (\%) & $225(42.3)$ & $157(38.3)$ & 0.24 \\
$\quad$ Ulcerative colitis & $307(57.3)$ & $253(61.7)$ & \\
Crohn's disease & $43(8)$ & $44(10.7)$ & 0.2 \\
New IBD cases, N (\%) & & & \\
\hline
\end{tabular}

IBD: inflammatory bowel disease; NA: not assessed; SD: standard deviation; 
mean age of admitted IBD patients was significantly lower in 2020 in comparison to the corresponding 2019-time frame $(\mathrm{p}=0.006)$. During the study period, 44 new IBD cases were admitted to our Tertiary Care Unit in 2020 in comparison to 43 cases in 2019, with no significant differences in mean age, gender and IBD phenotype distributions.

The number of endoscopic evaluations for IBD patients was 3 -fold reduced, from 237 procedures in 2019 to 84 in the corresponding period of 2020. The comparison of IBD case management between the two study groups is presented in Table II.

Table II. Comparison of IBD therapy during the two study periods (2019 vs 2020).

\begin{tabular}{lccc}
\hline Treatment, N (\%) & 2019 & 2020 & $\mathrm{p}$ \\
\hline 5-aminosalicylic acid & $219(41.2)$ & $313(29)$ & 0.0001 \\
Azathioprine & $125(23.6)$ & $86(20.9)$ & 0.38 \\
Topical corticosteroids & $19(3.6)$ & $11(2.7)$ & 0.74 \\
Systemic corticosteroids & $36(6.7)$ & $28(6.8)$ & \\
Biologicals & $308(57.9)$ & $326(79.5)$ & $<0.0001$ \\
Combo therapy & $66(12.4)$ & $69(16.8)$ & 0.054 \\
(Azathioprine + Biological) & & & \\
Surgery & $12(2.3)$ & $14(3.4)$ & 0.38 \\
\hline
\end{tabular}

A significantly lower proportion of admitted patients was managed with 5-aminosalicylic acid (5-ASA) whereas a substantially higher number of patients received biological therapy in 2020 in comparison to the corresponding 2019time frame. There was no significant difference between the percentage of patients receiving azathioprine (AZA), corticosteroid treatment or in the surgery referral of patients. There was a marginally significant increase in cases receiving Combo therapy in the 2020 study group.

Overall, only 34 (6.4\%) of IBD cases required the initiation of a biological agent due to a disease flare-up in the 2019 referral period vs. $49(12 \%)$ of patients in the corresponding 2020 -time frame, $(p=0.004)$. The distribution of the biological agent used in patients already on biological therapy was significantly different in 2019 in comparison to 2020 period mainly due to a decrease in adalimumab and an increase in vedolizumab prescription ( $\mathrm{p}=0.0004)$ : adalimumab $(131 / 48.8 \%$ vs $114 / 41 \%)$, infliximab (135/50.4\% vs $142 / 51 \%)$, vedolizumab
( $2 / 0.7 \%$ vs $17 / 6.1 \%)$, other biologicals ( $0 / 0 \%$ vs $5 / 1.8 \%)$. In patients with an initiation of a biological agent, there was also a statistically significant difference in the distribution of the biological agent used $(\mathrm{p}=0.0001)$ : adalimumab $(13 / 38.2 \%$ vs $8 / 17.4 \%)$, infliximab $(21 / 61.8 \%$ vs $18 / 39.1 \%)$, vedolizumab ( $0 / 0 \%$ vs $19 / 41.3 \%)$, other biologicals $(0 / 0 \%$ vs $1 / 2 \%)$. In $2019,14(4.6 \%)$ of hospitalized patients already on biologicals required treatment optimization or a switch of biologicals, in comparison to 36 (11\%) of patients in 2020 ( $\mathrm{p}=0.004)$. In 2019 $11.4 \%$ of patients under biological therapy received a biosimilar agent in comparison to $16.8 \%$ in $2020(\mathrm{p}=0.09)$. In patients with a biological agent initiation in 2019, 29.4\% used a biosimilar in comparison to $19.2 \%$ in the 2020 referral period $(\mathrm{p}=0.54)$.

During the studied period in 2020, 7 patients (1.7\%) were diagnosed with SARS-Cov2 infection. All patients presented mild symptoms, with fever, anosmia, cough, and diarrhea (Table III). Two of the patients were on adalimumab and 3 patients on infliximab (one with combo therapy with AZA). All patients continued the biological IBD treatment after a two-week delay, without any interruptions or modifications of scheduled doses since the COVID-19 infection. One ulcerative colitis (UC) patient recently initiated biological therapy with Vedolizumab prior to COVID-19 and still had gastrointestinal symptoms and active disease at COVID-19 onset. Another patient was newly diagnosed with UC and received glucocorticoids treatment for a severe flare, having a good clinical response that allowed tapering of steroids and continuing only with 5-ASA treatment. Two patients were referred to COVID-19 support hospitals for treatment and had an uneventful recovery from viral infection while continuing specific IBD treatment. No severe COVID-19 disease was recorded in our IBD patients during the referral period.

Treatment adherence during the referral period in 2020 was also evaluated, with a special focus on biological therapy. 264 patients $(86.8 \%)$ on biological treatment respected their treatment schedule and all patients with subcutaneous treatment were adherent to the treatment schedule; 20 patients (6.6\%) on infliximab skipped or postponed one infusion and 15 cases $(4.9 \%)$ received their treatment from another (primary or secondary) care unit. Three patients (1.0\%) were non-compliant in attending physician counselling to continue the treatment during the 2020 referral period without interruptions: two patients on infliximab and one patient on vedolizumab. These

Table III. IBD patient's characteristics at diagnosis of COVID-19.

\begin{tabular}{|c|c|c|c|c|c|c|c|}
\hline $\begin{array}{l}\text { Case } \\
\text { No. }\end{array}$ & Gender & Age & $\begin{array}{c}\text { IBD } \\
\text { phenotype }\end{array}$ & IBD severity & IBD treatment & $\begin{array}{l}\text { COVID-19 } \\
\text { severity }\end{array}$ & $\begin{array}{l}\text { Referral to COVID-19 } \\
\text { support hospital }^{*}\end{array}$ \\
\hline 1 & female & 51 & $\mathrm{UC}$ & severe active disease & Vedolizumab & Mild & Yes \\
\hline 2 & male & 47 & UC & clinical remission & Adalimumab & Mild & No \\
\hline 3 & male & 19 & UC & severe active disease & Corticosteroids & Mild & Yes \\
\hline 4 & male & 70 & $\mathrm{CD}$ & moderate disease & Infliximab & Mild & No \\
\hline 5 & male & 52 & UC & moderate disease & Infliximab & Mild & No \\
\hline 6 & female & 35 & $\mathrm{CD}$ & clinical remission & $\begin{array}{l}\text { Azathioprine }+ \\
\text { Infliximab }\end{array}$ & Mild & No \\
\hline 7 & male & 38 & CD & mild disease & Adalimumab & Mild & No \\
\hline
\end{tabular}

CD: Crohn's disease; COVID-19: coronavirus disease 2019; IBD: inflammatory bowel disease; UC: ulcerative colitis; ${ }^{*}$ with dedicated gastroenterology ward 
patients subsequently resumed their biological treatment after they missed two infusions, however without disease flare-up.

\section{DISCUSSION}

The occurrence of SARS-Cov2 pandemic has changed the management of IBD patients in our referral center, as has happened in other centers dedicated to IBD care in the world. In 2020, compared with pre-pandemic practice, we managed to reduce the hospitalization time, and, as consequence, a significantly higher proportion of patients received all the medical care they needed in one day hospitalization setting. The number of new IBD cases admitted to hospital was similar between the two periods, reflecting unchanged referral and hospitalization policies for potentially severe clinical situations as well as timely diagnosis of new cases.

Analyzing the treatments received by the hospitalized patients, we documented a significantly reduced prescription of 5-ASA, most probably resulting from lower rates of admission for patients with mild flares of disease, a group of patients that could be safely managed by telemedicine, utilizing surrogate markers of inflammation (e.g. calprotectin). The great majority of patients admitted to our center received biological therapy (nearly $80 \%$ ), significantly more in 2020 compared to 2019. Several causes contributed to this situation: hospital admissions were in line with the National Protocol for IBD Management that could not be postponed, since only tertiary gastroenterology centers were allowed to decide the continuation, halting or changing of a biologic therapy in IBD patients in Romania. A significantly larger number of patients required dose adjustments or switch to another agent in 2020 compared to 2019. Also, an increased number of patients initiated biologic therapy in the pandemic period (again significantly more than in the previous year). One explanation could be that physicians were reluctant to start corticosteroids in patients with moderate to severe flares of disease due to the risk of negative impact on the SARS-Cov2 viral infection. The International Organization for the Study of Inflammatory Bowel Diseases (IOIBD) stated that prednisone at doses over $20 \mathrm{mg} /$ day increases the risk of infection with SARS-Cov2 and also increases the risk of COVID-19 and recommended to reduce the doses or to the discontinuation of therapy [7]. In this context, in the attempt to control disease flares, biological agents were the available alternative. The risk/benefit clinical judgment prevailed also in the choice of biological therapy that was initiated. We identified a significantly higher number of vedolizumab prescriptions in 2020. We must emphasize that in Romania vedolizumab is available as a reimbursed biologic treatment for IBD only since December 2019, but even so, we consider the high number of vedolizumab prescription significant. This could relate to the safety profile of vedolizumab, based on the reported small risk for infections of this gut selective anti-integrin agent $[8,9]$.

Integrins are a family of cell surface receptors that mediate cellular interactions, including viral attachment. There are reports in the literature suggesting that SARS-Cov2 spike protein $S$ (the main glycoprotein present at the surface of the virion involved in host receptor binding) has also arginineglycine-aspartate (RGD) integrin binding motif, besides the well-established angiotensin converting enzyme 2 (ACE2) binding motif, that facilitates adhesion, endocytosis and infection of different target cells $[10,11]$. One can speculate on a possible antiviral effect for agents that block integrin binding (anti-integrins), and further research is of value in this field. Reported outcomes of patients with SARS-Cov2 infection that are receiving treatment with vedolizumab for IBD derived from large international databases (such as SECURE-IBD) could be particularly useful in this regard.

It has been reported that patients with IBD have no greater risk of SARS-CoV-2 infection than the general population [12]. In our cohort, during the referral period, only 7 patients were positive for SARS-Cov2-infection, five on anti-tumor necrosis factor alpha ( 3 on infliximab and 2 on adalimumab), one on vedolizumab and one received corticosteroids (newly diagnosed ulcerative colitis, negative PCR at admission). All patients had mild forms of the viral infection. Patients with IBD from Nancy and Milan cohorts also showed mild/ moderate forms of SARS-Cov2 infection, only one third being hospitalized, with no intensive care unit admissions and no deaths [12]. Results from the largest database of IBD patients infected with SARS-Cov2, SECURE-IBD, show that from almost 2,800 reported cases, 23\% IBD patients needed to be hospitalized, $4 \%$ in the intensive care units, $3 \%$ needed invasive respiratory support, and there were $2 \%$ reported deaths [13]. Since COVID-19 mortality rate is different between countries, ranging in Europe between 0.8 and 7.6\% [14] and it is influenced by many co-factors (age, comorbidities), it is difficult to draw a conclusion regarding increased or reduced risk of severe outcomes for IBD patients with COVID-19. In the pandemic scenario, with sometimes conflicting reports, the patient perceived the risk of an unfavorable outcome is important for the adherence to IBD treatment. A web survey realized with the support of European Federation of Crohn's and Ulcerative Colitis Associations (EFCCA) that investigated concerns, fears and behaviors of patients with IBD during the pandemic showed that nearly two thirds of responders stated that immunosuppressive drugs were associated with a higher risk of viral infection but a large part $(88 \%)$ of them did not want to discontinue IBD medication and almost all (96\%) had not stopped taking the medication on their own [15]. In our study group, most of the IBD patients respected their treatment schedule $(86.8 \%)$. We must emphasize that all patients with subcutaneous treatment were strictly adherent, while only a small percentage $(6.6 \%)$ of patients with intravenous infusions postponed for longer or shorter intervals the drug administration. This rises the question whether, especially during epidemic periods, we should favor the initiation of ambulatory treatments (subcutaneous or oral), with a positive impact on patient's adherence.

\section{CONCLUSIONS}

The SARS-Cov2 pandemic has changed the way IBD patients were managed in our Gastroenterology Tertiary Referral Center, with a new focus on telemedicine for mild flares of disease, and optimized medical treatment to maximize the benefit/risk ratio, in the context of the major viral outbreak of COVID-19. However, incidence of SARS-Cov2 infection 
during the first wave of COVID-19 infection in IBD patients referred to our Gastroenterology Unit was low, presented only with mild symptoms and did not adversely impact the IBD disease course.

\section{Conflicts of interest: None to declare.}

Authors' contribution: R.V., R.I. and C.G. conceived and designed the study. R.C., T.S., I.S., S.I. collected the data. S.I. and R.I. performed the statistical analysis. R.V., R.I., S.I. and R.C. interpreted the data and drafted the manuscript. L.G., M.D. and C.G. revised the manuscript and approved the final version to be published.

\section{REFERENCES}

1. Covid-19 coronavirus pandemic. Available at:https://www. worldometers.info/coronavirus/. Accessed: 29 November 2020.

2. Săftoiu A, Tomulescu V, Tanţău M, et al. SRED-ARCE Recommendations for Minimally Invasive Interventions During the COVID-19 Pandemic in Romania. Chirurgia (Bucur) 2020;115:289-306. doi:10.21614/ chirurgia.115.3.289

3. Elli L, Tontini GE, Scaramella L, et al. Reopening Endoscopy after the COVID-19 Outbreak: Indications from a High Incidence Scenario. J Gastrointestin Liver Dis 2020;29:295-299. doi:10.15403/jgld-2687

4. Gheorghe C. Clinical Insights for Gastroenterology and Hepatology Providers during the COVID-19 Pandemic. J Gastrointest Liver Dis 2020;29:131-133. doi:10.15403/jgld-2603

5. Al-Ani AH, Prentice RE, Rentsch CA, et al. Review article: prevention, diagnosis and management of COVID-19 in the IBD patient. Aliment Pharmacol Ther 2020;52:54-72. doi:10.1111/apt.15779

6. Silvio D, Cecconi M, Spinelli A. Management of IBD during the COVID-19 outbreak: resetting clinical priorities. Nat Rev Gastroenterol Hepatol 2020;17:253-255. doi:10.1038/s41575-020-0294-8
7. Rubin DT, Abreu MT, Rai V, Siegel CA; International Organization for the Study of Inflammatory Bowel Disease. Management of patients with Crohn's Disease and Ulcerative colitis during the Coronavirus Disase-2019 pandemic: results of an international meeting. Gastroenterology 2020;159:6-13. doi:10.1053/j.gastro.2020.04.002

8. Ng SG, Hilmi IN, Blake A, et al. Low frequency of opportunistic infections in patients receiving Vedolizumab in clinical trials and post-marketing. Inflamm Bowel Dis 2018;24:2431-2441. doi:10.1093/ $\mathrm{ibd} / \mathrm{izy} 153$

9. Meserve J, Aniwan S, Koliani-Pace JL, et al. Retrospective analysis of safety of Vedolizumab in patients with Inflammatory Bowel Diseases. Clin Gastroenterol Hepatol 2019;17:1533-1540.e2. doi:10.1016/j. cgh.2018.09.035

10. Sigrist CJ, Bridge A, LeMercier P. A potential role for integrins in host cell entry by SARS-CoV-2. Antiviral Res 2020;177:104759. doi:10.1016/j.antiviral.2020.104759

11. Tresoldi I, Sangiulo CF, Manzari V, Modesti A. SARS-CoV-2 and infectivity: possible increase in infectivity associated to integrin motif expression. J Med Virol 2020;92:1741-1742. doi:10.1002/jmv.25831

12. Alloca M, Fiorino G, Zallot $\mathrm{C}$, et al. Incidence, and patterns of COVID 19 among inflammatory bowel disease patients from Nancy and Milan cohorts. Clin Gastroenterol Hepatol 2020;18:2134-2135. doi:10.1016/j. cgh.2020.04.071

13. Brenner EJ, Ungaro RC, Colombel JF, Kappelman MD. SECURE-IBD Database Public Data Update. Available at: https://covidibd.org/. Accessed:01/11/2020.

14. European Centre for Disease Prevention and Control. Daily update of new reported cases of COVID-19 by country worldwide. Available at:https://www.ecdc.europa.eu/en/publications-data/download-todaysdata-geographic-distribution-covid-19-cases-worldwide

15. D’Amico F, Rahier JF, Leone S, Peyrin-Biroulet L, Danese S. Views of patients with inflammatory bowel disease on the COVID-19 pandemic: a global survey. Lancet Gastroenterol Hepatol 2020;5:631-632. doi:10.1016/S2468-1253(20)30151-5 